PROCEEDINGS OF THE

AMERICAN MATHEMATICAL SOCIETY

Volume 131, Number 9, Pages 2681-2692

S 0002-9939(02)06892-2

Article electronically published on December 30, 2002

\title{
HOWE DUALITY AND THE QUANTUM GENERAL LINEAR GROUP
}

\author{
R. B. ZHANG
}

(Communicated by Dan M. Barbasch)

\begin{abstract}
A Howe duality is established for a pair of quantized enveloping algebras of general linear algebras. It is also shown that this quantum Howe duality implies Jimbo's duality between $\mathrm{U}_{q}\left(\mathfrak{g l}_{n}\right)$ and the Hecke algebra.
\end{abstract}

\section{INTRODUCTION}

A treatment of classical invariant theory based on the highest weight theory of representations of complex reductive algebraic groups was given in [Ho], where the organizing principle is the multiplicity free actions of reductive groups on algebraic varieties commonly referred to as Howe dualities. In the context of the general linear group over the complex field, the Howe duality amounts to the following result (or a variant of it in terms of right modules): Regard $\mathbb{C}^{k}$ as the natural (left) $\mathrm{GL}_{k}(\mathbb{C})$-module for each $k . \mathrm{GL}_{m}(\mathbb{C}) \times \mathrm{GL}_{n}(\mathbb{C})$ acts on the algebra of regular functions $\mathcal{P}\left(\mathbb{C}^{m} \otimes \mathbb{C}^{n}\right)$ on $\mathbb{C}^{m} \otimes \mathbb{C}^{n}$ in a multiplicity free manner. Moreover, $\mathcal{P}\left(\mathbb{C}^{m} \otimes \mathbb{C}^{n}\right)=\bigoplus_{\lambda \in \Lambda_{\min (m, n)}} L_{\lambda}^{[m], 0} \otimes L_{\lambda}^{[n], 0}$, where $L_{\lambda}^{[m], 0}$ (respectively $L_{\lambda}^{[n], 0}$ ) denotes the irreducible $\mathrm{GL}_{m}(\mathbb{C})$-module (respectively $\mathrm{GL}_{n}(\mathbb{C})$-module) with highest weight $\lambda$, and $\Lambda_{\min (m, n)}$ is the set of the highest weights corresponding to partitions of depth $\leq \min (m, n)$. While this result can be proven by relatively elementary means, its implications are far reaching. As shown in $[\mathrm{Ho}$, the First and Second Fundamental Theorems of the invariant theory for the general linear group are all immediate consequences of this result. In particular, the celebrated Schur duality can be easily derived from the Howe duality.

This paper aims to develop a quantum version of the Howe duality for a pair of quantized enveloping algebras of general linear algebras at generic $q$. The main problem is to construct a non-commutative analogue $\mathcal{V}_{m, n}$ of $\mathcal{P}\left(\mathbb{C}^{m} \otimes \mathbb{C}^{n}\right)$. We achieve this by investigating the Hopf algebra of functions on the quantum general linear group. With the $\mathcal{V}_{m, n}$ given in Definition 3.4, we prove

Theorem 1.1. $\mathcal{V}_{m, n}$ forms a module algebra under the left action of $\mathrm{U}_{q}\left(\mathfrak{g l}_{m}\right)$ and right action of $\mathrm{U}_{q}\left(\mathfrak{g l}_{n}\right)$, with the actions of the two quantum algebras being mutual centralizers. Furthermore,

$$
\mathcal{V}_{m, n}=\bigoplus_{\lambda \in \Lambda_{\min (m, n)}} L_{\lambda}^{[m]} \otimes \tilde{L}_{\lambda}^{[n]}
$$

Received by the editors June 24, 2001 and, in revised form, April 7, 2002.

2000 Mathematics Subject Classification. Primary 17B37, 20G42, 17B10.

(C)2002 American Mathematical Society 
where $L_{\lambda}^{[m]}$ (respectively $\tilde{L}_{\lambda}^{[n]}$ ) denote the irreducible left $\mathrm{U}_{q}\left(\mathfrak{g l}_{m}\right)$-module (respectively right $\mathrm{U}_{q}\left(\mathfrak{g l}_{n}\right)$-module) of type $I[\mathrm{CP}]$ with highest weight $\lambda$.

The notion of a module algebra over a bi-algebra is explained in Definition 3.1.

It is an old result of Jimbo's [Ji] dating back to the mid-1980s (and having since been treated by many other people) that there existed a duality between $\mathrm{U}_{q}\left(\mathfrak{g l}_{n}\right)$ and the Hecke algebra analogous to the celebrated Schur duality between $\mathrm{GL}_{n}(\mathbb{C})$ and the symmetric group. We shall derive the quantum Schur duality from the quantum Howe duality established here. For doing this, we investigate the representations of the $q$-Weyl group [KR] of $\mathrm{U}_{q}\left(\mathfrak{g l}_{n}\right)$ on the spaces of zero weight vectors (see equation (4.4)) of finite-dimensional $\mathrm{U}_{q}\left(\mathfrak{g l}_{n}\right)$-modules. They give rise to representations of the Hecke algebra, which we believe are of independent interest, thus are studied in some detail.

\section{The quantum General Linear Group}

Let $\mathfrak{h}^{*}$ be the complex vector space which has a basis $\left\{\epsilon_{a} \mid 1 \leq a \leq n\right\}$ and is endowed with a non-degenerate symmetric bilinear form $():, \mathfrak{h}^{*} \times \mathfrak{h}^{*} \rightarrow \mathbb{C}$ defined by $\left(\epsilon_{a}, \epsilon_{b}\right)=\delta_{a b}$. Set $\mathfrak{h}_{\mathbb{Z}}^{*}=\bigoplus_{a} \mathbb{Z} \epsilon_{a}$. The quantized enveloping algebra $\mathrm{U}_{q}\left(\mathfrak{g l}_{n}\right)$ of the general linear algebra $\mathfrak{g l}_{n}$ is a Hopf algebra over the field of rational functions $\mathbb{C}(t)$ in $t$. As a unital associative algebra, $\mathrm{U}_{q}\left(\mathfrak{g l}_{n}\right)$ is generated by $K_{a}, K_{a}^{-1}, 1 \leq$ $a \leq n$, and $E_{b}, F_{b}, 1 \leq b<n$, subject to the standard relations, which we spell out in order to fix our notation:

$$
\begin{aligned}
& K_{a} K_{a}^{-1}=1, K_{a}^{ \pm 1} K_{b}^{ \pm 1}=K_{b}^{ \pm 1} K_{a}^{ \pm 1}, \\
& K_{a} E_{b} K_{a}^{-1}=q^{\left(\epsilon_{b}-\epsilon_{b+1}, \epsilon_{a}\right)} E_{b}, K_{a} F_{b} K_{a}^{-1}=q^{-\left(\epsilon_{b}-\epsilon_{b+1}, \epsilon_{a}\right)} F_{b}, \\
& E_{a} F_{b}-F_{b} E_{a}=\delta_{a b}\left(K_{a} K_{a+1}^{-1}-K_{a}^{-1} K_{a+1}\right) /\left(q-q^{-1}\right), \\
& E_{a} E_{b}=E_{b} E_{a}, \quad F_{a} F_{b}=F_{b} F_{a}, \quad|a-b|>1, \\
& \mathcal{S}_{a}^{(+)}{ }_{a \pm 1}= \mathcal{S}_{a a \pm 1}^{(-)}=0,
\end{aligned}
$$

where $q=t^{2}$, and

$$
\begin{aligned}
& \mathcal{S}_{a a \pm 1}^{(+)}=\left(E_{a}\right)^{2} E_{a \pm 1}-\left(q+q^{-1}\right) E_{a} E_{a \pm 1} E_{a}+E_{a \pm 1}\left(E_{a}\right)^{2}, \\
& \mathcal{S}_{a a \pm 1}^{(-)}=\left(F_{a}\right)^{2} F_{a \pm 1}-\left(q+q^{-1}\right) F_{a} F_{a \pm 1} F_{a}+F_{a \pm 1}\left(F_{a}\right)^{2} .
\end{aligned}
$$

We will also need the explicit form of the other structural maps of $\mathrm{U}_{q}\left(\mathfrak{g l}_{n}\right)$ : the comultiplication $\Delta: \mathrm{U}_{q}\left(\mathfrak{g l}_{n}\right) \rightarrow \mathrm{U}_{q}\left(\mathfrak{g l}_{n}\right) \otimes \mathrm{U}_{q}\left(\mathfrak{g l}_{n}\right)$,

$$
\begin{aligned}
\Delta\left(E_{a}\right) & =E_{a} \otimes K_{a} K_{a+1}^{-1}+1 \otimes E_{a}, \\
\Delta\left(F_{a}\right) & =F_{a} \otimes 1+K_{a}^{-1} K_{a+1} \otimes F_{a}, \\
\Delta\left(K_{a}^{ \pm 1}\right) & =K_{a}^{ \pm 1} \otimes K_{a}^{ \pm 1}
\end{aligned}
$$

the co-unit $\epsilon: \mathrm{U}_{q}\left(\mathfrak{g l}_{n}\right) \rightarrow \mathbb{C}(t)$,

$$
\epsilon\left(E_{a}\right)=\epsilon\left(F_{a}\right)=0, \quad \epsilon\left(K_{b}^{ \pm 1}\right)=1 ;
$$

and the antipode $S: \mathrm{U}_{q}\left(\mathfrak{g l}_{n}\right) \rightarrow \mathrm{U}_{q}\left(\mathfrak{g l}_{n}\right)$,

$$
S\left(E_{a}\right)=-E_{a} K_{a}^{-1} K_{a+1}, \quad S\left(F_{a}\right)=-K_{a} K_{a+1}^{-1} F_{a}, \quad S\left(K_{a}^{ \pm 1}\right)=K_{a}^{\mp 1} .
$$

We shall denote by $L_{\lambda}$ the irreducible left $\mathrm{U}_{q}\left(\mathfrak{g l}_{n}\right)$-module of type I (in the sense of [CP]) with highest weight $\lambda \in \mathfrak{h}_{\mathbb{Z}}^{*}$. More explicitly, if $v_{+}$is the highest weight 
vector of $L_{\lambda}$, then

$$
\begin{aligned}
& E_{a} v_{+}=0, \quad 1 \leq a<n, \\
& K_{a} v_{+}=q^{\left(\epsilon_{a}, \lambda\right)} v_{+}, \forall a .
\end{aligned}
$$

Similarly, we denote by $\tilde{L}_{\lambda}, \lambda \in \mathfrak{h}_{\mathbb{Z}}^{*}$, the irreducible right $\mathrm{U}_{q}\left(\mathfrak{g l}_{n}\right)$-module which admits a unique 1 -dimensional subspace $\mathbb{C}(t) \tilde{v}_{+}$such that

$$
\begin{aligned}
& \tilde{v}_{+} F_{a}=0, \quad 1 \leq a<n, \\
& \tilde{v}_{+} K_{a}=q^{\left(\epsilon_{a}, \lambda\right)} \tilde{v}_{+}, \forall a .
\end{aligned}
$$

We shall call $\tilde{L}_{\lambda}$ the irreducible right $\mathrm{U}_{q}\left(\mathfrak{g l}_{n}\right)$-module of type I with highest weight $\lambda$.

Define $\Lambda_{n}:=\left\{\sum_{a=1}^{n} \lambda_{a} \epsilon_{a} \mid \lambda_{a} \in \mathbb{Z}_{+}, \lambda_{a} \geq \lambda_{a+1}\right\}$. Let $\Lambda_{k}=\Lambda_{n} \cap\left(\bigoplus_{i=1}^{k} \mathbb{Z} \epsilon_{i}\right)$ if and only if $k \leq n$. For $\lambda=\sum_{a=1}^{n} \lambda_{a} \epsilon_{a} \in \Lambda_{n}$, we set $|\lambda|=\sum_{a=1}^{n} \lambda_{a}$. Let $\Lambda_{n}(d)=\left\{\lambda \in \Lambda_{n}|| \lambda \mid=d\right\}$. The irreducible left (respectively right) $\mathrm{U}_{q}\left(\mathfrak{g l}_{n}\right)$-module $L_{\lambda}$ (respectively $\tilde{L}_{\lambda}$ ) is finite dimensional if $\lambda \in \Lambda_{n}$.

Of particular interest for us here is the contravariant vector module $V=L_{\epsilon_{1}}$. It has the standard basis $\left\{v_{a} \mid 1 \leq a \leq n\right\}$ with the action of $\mathrm{U}_{q}\left(\mathfrak{g l}_{n}\right)$ given by

$$
\begin{aligned}
E_{a} v_{b} & =\delta_{b a+1} v_{a}, \\
F_{a} v_{b} & =\delta_{b a} v_{a+1}, \\
K_{a} v_{b} & =\left[1+(q-1) \delta_{a b}\right] v_{b} .
\end{aligned}
$$

We shall denote by $\pi$ the irreducible $\mathrm{U}_{q}\left(\mathfrak{g l}_{n}\right)$-representation relative to this basis. The tensor power $V^{\otimes k}$ of $V$ is completely reducible for any $k \in \mathbb{Z}_{+}$:

$$
V^{\otimes k}=\bigoplus_{\lambda \in \Lambda_{n}(k)} m_{\lambda} L_{\lambda}
$$

where $0<m_{\lambda} \in \mathbb{Z}_{+}$is the multiplicity of $L_{\lambda}$.

Let $\mathrm{U}_{q}\left(\mathfrak{g l}_{n}\right)^{0}:=\left\{f \in \mathrm{U}_{q}\left(\mathfrak{g l}_{n}\right)^{*} \mid\right.$ ker $f$ contains a cofinite ideal of $\left.\mathrm{U}_{q}\left(\mathfrak{g l}_{n}\right)\right\}$ denote the finite dual of the quantized enveloping algebra $\mathrm{U}_{q}\left(\mathfrak{g l}_{n}\right)$. Standard Hopf algebra theory asserts [Mon] that $\mathrm{U}_{q}\left(\mathfrak{g l}_{n}\right)^{0}$ has the structure of a Hopf algebra induced by the Hopf algebra structure of $\mathrm{U}_{q}\left(\mathfrak{g l}_{n}\right)$. Denote by $m_{0}, \Delta_{0}, \epsilon_{0}$, and $S_{0}$ the multiplication, comultiplication, co-unit and antipode of $\mathrm{U}_{q}\left(\mathfrak{g l}_{n}\right)^{0}$ respectively.

Let $t_{a b}, 1 \leq a, b \leq n$, be the matrix elements of the contravariant vector representation of $\mathrm{U}_{q}\left(\mathfrak{g l}_{n}\right)$ relative to the standard basis. That is, the $t_{a b}$ are elements of the dual space $\mathrm{U}_{q}\left(\mathfrak{g l}_{n}\right)^{*}$ of $\mathrm{U}_{q}\left(\mathfrak{g l}_{n}\right)$ defined by

$$
x v_{a}=\sum_{b}\left\langle t_{b a}, x\right\rangle v_{b}, \forall x \in \mathrm{U}_{q}\left(\mathfrak{g l}_{n}\right),
$$

where $\langle\cdot, \cdot\rangle$ represents dual space pairing. Clearly the matrix elements of any finite-dimensional representation of $\mathrm{U}_{q}\left(\mathfrak{g l}_{n}\right)$ belong to $\mathrm{U}_{q}\left(\mathfrak{g l}_{n}\right)^{0}$. In particular, $t_{a b} \in$ $\mathrm{U}_{q}\left(\mathfrak{g l}_{n}\right)^{0}, \forall a, b$. Under the co-multiplication of $\mathrm{U}_{q}\left(\mathfrak{g l}_{n}\right)^{0}$, we have

$$
\Delta_{0}\left(t_{a b}\right)=\sum_{c} t_{a c} \otimes t_{c b}
$$

Definition 2.1. Denote by $\mathcal{T}_{q}\left(\mathfrak{g l}_{n}\right)$ the subbialgebra of $\mathrm{U}_{q}\left(\mathfrak{g l}_{n}\right)^{0}$ generated by the $t_{a b}, 1 \leq a, b \leq n$. 
Any finite-dimensional left $\mathrm{U}_{q}\left(\mathfrak{g l}_{n}\right)$-module has a natural right $\mathrm{U}_{q}\left(\mathfrak{g l}_{n}\right)^{0}$-comodule structure. In particular, if $\left\{w_{\alpha}^{(\lambda)} \mid \alpha=1,2, \ldots, \operatorname{dim} L_{\lambda}\right\}$ is any basis of the finite-dimensional irreducible $\mathrm{U}_{q}\left(\mathfrak{g l}_{n}\right)$-module $L_{\lambda}$, then the right $\mathrm{U}_{q}\left(\mathfrak{g l}_{n}\right)^{0}$-comodule action $L_{\lambda} \rightarrow L_{\lambda} \otimes \mathrm{U}_{q}\left(\mathfrak{g l}_{n}\right)^{0}$ is given by $w_{\alpha}^{(\lambda)} \mapsto \sum_{\beta} w_{\beta}^{(\lambda)} \otimes t_{\beta \alpha}^{(\lambda)}$, where the $t_{\beta \alpha}^{(\lambda)}$ are the matrix elements of the $\mathrm{U}_{q}\left(\mathfrak{g l}_{n}\right)$-representation associated to $L_{\lambda}$ relative to the given basis. If $\lambda \in \Lambda_{n}$, then it follows from (2.1) that all the $t_{\alpha \beta}^{(\lambda)}$ belong to $\mathcal{T}_{q}\left(\mathfrak{g l}_{n}\right)$. Furthermore, Burnside's theorem implies that the matrix elements of all the irreducible representations of $\mathrm{U}_{q}\left(\mathfrak{g l}_{n}\right)$ with highest weights in $\Lambda_{n}$ are linearly independent. Thus

Proposition 2.1. $\left\{t_{\beta \alpha}^{(\lambda)} \mid 1 \leq \alpha, \beta \leq \operatorname{dim} L_{\lambda}, \lambda \in \Lambda_{n}\right\}$ forms a basis of $\mathcal{T}_{q}\left(\mathfrak{g l}_{n}\right)$.

This will be called a Peter-Weyl basis of $\mathcal{T}_{q}\left(\mathfrak{g l}_{n}\right)$.

Below we give a more explicit description of the bialgebra $\mathcal{T}_{q}\left(\mathfrak{g l}_{n}\right)$ in terms of generators and relations following [FRT, Ta]. Let $R$ be the $R$-matrix associated with the irreducible $\mathrm{U}_{q}\left(\mathfrak{g l}_{n}\right)$-representation $\pi$ furnished by the module $V$ in the standard basis:

$$
R:=1 \otimes 1+\sum_{a=1}^{n}(q-1) e_{a a} \otimes e_{a a}+\left(q-q^{-1}\right) \sum_{a<b} e_{a b} \otimes e_{b a} .
$$

$R$ satisfies the quantum Yang-Baxter equation, and also the following commutation relation:

$$
R(\pi \otimes \pi) \Delta(x)=(\pi \otimes \pi) \Delta^{\prime}(x) R, \forall x \in \mathrm{U}_{q}\left(\mathfrak{g l}_{n}\right),
$$

where $\Delta^{\prime}$ is the opposite co-multiplication of $\mathrm{U}_{q}\left(\mathfrak{g l}_{n}\right)$.

Definition 2.2 ([FRT $)$. Let $\mathbb{C}_{q}\left[X_{a b}\right]$ be the unital associative algebra over $\mathbb{C}(t)$ generated by $X_{a b}, 1 \leq a, b \leq n$, subject to the following relations:

$$
R_{12} X_{1} X_{2}=X_{2} X_{1} R_{12},
$$

where $R_{12}=R \otimes 1, X_{1}=\sum_{a, b} e_{a b} \otimes 1 \otimes X_{a b}, X_{2}=\sum_{a, b} 1 \otimes e_{a b} \otimes X_{a b}$.

As is well known, $\mathbb{C}_{q}\left[X_{a b}\right]$ has the structure of a bialgebra, with the comultiplication $\Delta_{0}\left(X_{a b}\right)=\sum_{c} X_{a c} \otimes X_{c b}$, and co-unit $\epsilon_{0}\left(X_{a b}\right)=\delta_{a b}$.

Introduce an order $>$ for the pairs $(a, b), 1 \leq a, b \leq n$, such that $(a, b)>(a+k, c)$, $(a, b+k)>(a, b)$, if $k$ is a positive integer. Let $X^{(\mathbf{k})}=\prod_{a, b}^{>}\left(X_{a b}\right)^{k_{a b}}$, where the product is arranged according to the order $>$ of the indices of $X$ 's in such a way that $X_{a b}$ is positioned in front of $X_{c d}$ if $(a, b)>(c, d)$. The $\mathbf{k} \in \mathbb{Z}_{+}^{n^{2}}$ appearing in the superscript of $X^{(\mathbf{k})}$ denotes the square matrix $\left(k_{a b}\right)_{a, b=1}^{n}$. Set $|\mathbf{k}|=\sum_{a b} k_{a b}$. The following lemma is well known. It is an immediate consequence of the defining relations (2.4).

Lemma 2.1. The monomials $X^{(\mathbf{k})}, \mathbf{k} \in \mathbb{Z}_{+}^{n^{2}}$, form a basis of $\mathbb{C}_{q}\left[X_{a b}\right]$.

The following result was established in $\mathrm{Ta}$ :

Theorem 2.1 ( $[\mathrm{Ta}]$ ). The following map defines a bialgebra isomorphism:

$$
\imath: \mathbb{C}_{q}\left[X_{a b}\right] \rightarrow \mathcal{T}_{q}\left(\mathfrak{g l}_{n}\right), \quad X^{(\mathbf{k})} \mapsto T^{(\mathbf{k})}:=\prod_{a, b}^{>}\left(t_{a b}\right)^{k_{a b}} .
$$




\section{Multiplicity fRee ACtions}

Let $\Theta=\{1,2, \ldots, m\}, m \leq n$. We consider a number of Hopf subalgebras of $\mathrm{U}_{q}\left(\mathfrak{g l}_{n}\right)$ related to $m$. One is $\mathrm{U}_{q}\left(\mathfrak{g l}_{m}\right)$ generated by the elements of the following set: $\mathcal{S}=\left\{K_{i}^{ \pm 1}, 1 \leq i \leq m ; E_{j}, F_{j}, 1 \leq j<m\right\}$. We also have two parabolic Hopf subalgebras $\mathrm{U}_{q}\left(\mathfrak{p}_{+}\right)$and $\mathrm{U}_{q}\left(\mathfrak{p}_{-}\right)$, where $\mathrm{U}_{q}\left(\mathfrak{p}_{+}\right)$is generated by the elements of $\mathcal{S} \cup\left\{E_{\mu-1}, K_{\mu}^{ \pm 1}, \mu>m\right\}$, and $\mathrm{U}_{q}\left(\mathfrak{p}_{-}\right)$by the elements of $\mathcal{S} \cup\left\{F_{\mu-1}, K_{\mu}^{ \pm 1}, \mu>m\right\}$. Both Hopf subalgebras are graded by the Abelian group $\Gamma=\bigoplus_{\mu>m} \mathbb{Z}_{+}\left(\epsilon_{\mu-1}-\epsilon_{\mu}\right)$ :

$$
\begin{aligned}
& \mathrm{U}_{q}\left(\mathfrak{p}_{+}\right)=\bigoplus_{\beta \in \Gamma} \mathrm{U}_{q}\left(\mathfrak{p}_{+}\right)_{(\beta)}, \\
& \mathrm{U}_{q}\left(\mathfrak{p}_{-}\right)=\bigoplus_{\beta \in \Gamma} \mathrm{U}_{q}\left(\mathfrak{p}_{-}\right)_{(-\beta)} .
\end{aligned}
$$

Set $\mathrm{U}_{q}\left(\mathfrak{p}_{+}\right)^{+}=\bigoplus_{0 \neq \beta \in \Gamma} \mathrm{U}_{q}\left(\mathfrak{p}_{+}\right)_{(\beta)}$ and $\mathrm{U}_{q}\left(\mathfrak{p}_{-}\right)^{-}=\bigoplus_{0 \neq \beta \in \Gamma} \mathrm{U}_{q}\left(\mathfrak{p}_{-}\right)_{(-\beta)}$.

There exist natural actions of $\mathrm{U}_{q}\left(\mathfrak{g l}_{n}\right)$ on $\mathcal{T}_{q}\left(\mathfrak{g l}_{n}\right)$ which preserve the algebraic structure of $\mathcal{T}_{q}\left(\mathfrak{g l}_{n}\right)$ in the sense that the multiplication and unit of $\mathcal{T}_{q}\left(\mathfrak{g l}_{n}\right)$ are $\mathrm{U}_{q}\left(\mathfrak{g l}_{n}\right)$-module homomorphisms. Such actions are best described by the notion of module algebras over bi-algebras, which we recall presently.

Definition 3.1 ([Mon] $)$. Let $H$ be a bi-algebra. An associative algebra $A$ is called a left module algebra over $H$ if

(1) the underlying vector space of $A$ is a left $H$-module, and

(2) the multiplication $m: A \otimes A \rightarrow A$ and unit $\mathbb{1}_{A}: \mathbb{C} \rightarrow A$ of $A$ are $H$-module homomorphisms,

where the $H$-module structure of $A \otimes A$ is defined with respect to the comultiplication of $H$, and $\mathbb{C}$ is regarded as the $H$-module associated to the 1-dimensional representation given by the co-unit of $H$.

A left module algebra over $H^{\text {opp }}$ is also called a right module algebra over $H$, where $H^{\mathrm{opp}}$ denotes the bialgebra which has the same coalgebraic structure but the opposite algebraic structure to that of $H$.

It is easy to show that $\mathcal{T}_{q}\left(\mathfrak{g l}_{n}\right)$ forms a left module algebra over $\mathrm{U}_{q}\left(\mathfrak{g l}_{n}\right)$ under the following left action:

$$
\begin{aligned}
\Phi: \mathrm{U}_{q}\left(\mathfrak{g l}_{n}\right) \otimes \mathcal{T}_{q}\left(\mathfrak{g l}_{n}\right) & \rightarrow \mathcal{T}_{q}\left(\mathfrak{g l}_{n}\right), \\
x \otimes f & \mapsto \sum_{(f)} f_{(1)}\left\langle f_{(2)}, x\right\rangle,
\end{aligned}
$$

where we have used Sweedler's notation for the comultiplication of $f \in \mathcal{T}_{q}\left(\mathfrak{g l}_{n}\right)$ : $\Delta(f)=\sum_{(f)} f_{(1)} \otimes f_{(2)}$. Similarly, under the following right action:

$$
\begin{aligned}
\tilde{\Psi}: \mathcal{T}_{q}\left(\mathfrak{g l}_{n}\right) \otimes \mathrm{U}_{q}\left(\mathfrak{g l}_{n}\right) & \rightarrow \mathcal{T}_{q}\left(\mathfrak{g l}_{n}\right), \\
f \otimes x & \mapsto \sum_{(f)}\left\langle f_{(1)}, x\right\rangle f_{(2)},
\end{aligned}
$$

$\mathcal{T}_{q}\left(\mathfrak{g l}_{n}\right)$ forms a right module algebra over $\mathrm{U}_{q}\left(\mathfrak{g l}_{n}\right)$. Closely related to $\tilde{\Psi}$ is the following left $\mathrm{U}_{q}\left(\mathfrak{g l}_{n}\right)$-action on $\mathcal{T}_{q}\left(\mathfrak{g l}_{n}\right)$ :

$$
\begin{aligned}
\Psi: \mathrm{U}_{q}\left(\mathfrak{g l}_{n}\right) \otimes \mathcal{T}_{q}\left(\mathfrak{g l}_{n}\right) & \rightarrow \mathcal{T}_{q}\left(\mathfrak{g l}_{n}\right), \\
x \otimes f & \mapsto \sum_{(f)}\left\langle f_{(1)}, S(x)\right\rangle f_{(2)} .
\end{aligned}
$$


$\mathcal{T}_{q}\left(\mathfrak{g l}_{n}\right)$ forms a left $\mathrm{U}_{q}\left(\mathfrak{g l}_{n}\right)$-module algebra under $\Psi$ with respect to the opposite comultiplication of $\mathrm{U}_{q}\left(\mathfrak{g l}_{n}\right)$. It is obvious but important to observe that the action $\Phi$ commutes with $\Psi$ and $\tilde{\Psi}$. Also these actions naturally restrict to actions of the subalgebras $\mathrm{U}_{q}\left(\mathfrak{g l}_{m}\right), \mathrm{U}_{q}\left(\mathfrak{p}_{+}\right)$and $\mathrm{U}_{q}\left(\mathfrak{p}_{-}\right)$on $\mathcal{T}_{q}\left(\mathfrak{g l}_{n}\right)$.

Below we shall use the following notation. For any $f \in \mathcal{T}_{q}\left(\mathfrak{g l}_{n}\right), x \in \mathrm{U}_{q}\left(\mathfrak{g l}_{n}\right)$,

$$
\Psi_{x}(f):=\Psi(x \otimes f), \quad \Phi_{x}(f):=\Phi(x \otimes f), \quad \tilde{\Psi}_{x}(f):=\tilde{\Psi}(f \otimes x) .
$$

\section{Definition 3.2.}

$$
\mathcal{R}_{m}^{[n]}:=\left\{f \in \mathcal{T}_{q}\left(\mathfrak{g l}_{n}\right) \mid \Psi_{K_{c}^{ \pm 1}}(f)=f, c>m, \Psi_{x}(f)=0, x \in \mathrm{U}_{q}\left(\mathfrak{p}_{-}\right)^{-}\right\} .
$$

Lemma 3.1. The following set forms a basis of $\mathcal{R}_{m}^{[n]}$ :

$$
\left\{\prod_{1 \leq i \leq m, 1 \leq b \leq n}^{>}\left(t_{i b}\right)^{k_{i b}} \mid k_{i b} \in \mathbb{Z}_{+}\right\}
$$

Proof. Observe that $T^{(\mathbf{k})}=\imath\left(X^{(\mathbf{k})}\right) \in \mathcal{R}_{m}^{[n]}$ if and only if it belongs to this set. Now the lemma follows from Theorem 2.1

Theorem 3.1. (1) $\mathcal{R}_{m}^{[n]}$ forms a left module algebra over $\mathrm{U}_{q}\left(\mathfrak{g l}_{n}\right)$ under the action $\Phi$, and also forms a right module algebra over $\mathrm{U}_{q}\left(\mathfrak{g l}_{m}\right)$ under the action $\tilde{\Psi}$.

(2) $\Phi\left(\mathrm{U}_{q}\left(\mathfrak{g l}_{n}\right)\right)$ and $\tilde{\Psi}\left(\mathrm{U}_{q}\left(\mathfrak{g l}_{m}\right)\right)$ are mutual centralizers in $\operatorname{End}_{\mathbb{C}(t)}\left(\mathcal{R}_{m}^{[n]}\right)$. Furthermore, with respect to the joint action $\Phi \otimes \tilde{\Psi}$ of $\mathrm{U}_{q}\left(\mathfrak{g l}_{n}\right) \otimes \mathrm{U}_{q}\left(\mathfrak{g l}_{n}\right)$,

$$
\mathcal{R}_{m}^{[n]} \cong \bigoplus_{\lambda \in \Lambda_{m}} L_{\lambda} \otimes \tilde{L}_{\lambda}^{(0)},
$$

where $\tilde{L}_{\lambda}^{(0)}$ denotes the irreducible right $\mathrm{U}_{q}\left(\mathfrak{g l}_{m}\right)$-module with highest weight $\lambda \in \Lambda_{m}$.

Proof. Consider part (1) first. Let

$$
\mathcal{C}^{-}:=\left(\bigoplus_{a>m}\left[\mathbb{C}(t)\left(K_{a}-1\right) \oplus \mathbb{C}(t)\left(K_{a}^{-1}-1\right)\right]\right) \bigoplus \mathrm{U}_{q}\left(\mathfrak{p}_{-}\right)^{-},
$$

which forms a two-sided co-ideal of $\mathrm{U}_{q}\left(\mathfrak{g l}_{n}\right)$. For any $f, g \in \mathcal{R}_{m}^{[n]}$,

$$
\Psi_{x}(f g)=\sum_{(x)} \Psi_{x_{(1)}}(f) \Psi_{x_{(2)}}(g)=0, \forall x \in \mathcal{C}^{-} .
$$

Thus, $f g$ belongs to $\mathcal{R}_{m}^{[n]}$, that is, $\mathcal{R}_{m}^{[n]}$ forms a subalgebra of $\mathcal{T}_{q}\left(\mathfrak{g l}_{n}\right)$.

Since the left actions $\Phi$ and $\Psi$ commute, $\mathcal{R}_{m}^{[n]}$ clearly forms a left $\mathrm{U}_{q}\left(\mathfrak{g l}_{n}\right)$-module under $\Phi$. Consider $\tilde{\Psi}_{u}(f)=\sum\left\langle f_{(1)}, u\right\rangle f_{(2)}$ for $u \in \mathrm{U}_{q}\left(\mathfrak{g l}_{m}\right)$ and $f \in \mathcal{R}_{m}^{[n]}$. Now

$$
\begin{aligned}
\Psi_{K_{c}} \tilde{\Psi}_{u}(f) & =\tilde{\Psi}_{K_{c} u K_{c}^{-1}}(f)=\tilde{\Psi}_{u}(f), c>m, \\
\Psi_{x} \tilde{\Psi}_{u}(f) & =\Psi_{x S^{-1}(u)}(f)=0, x \in \mathrm{U}_{q}\left(\mathfrak{p}_{-}\right)^{-},
\end{aligned}
$$

where in the last equation we have used the fact that $x S^{-1}(u) \in \mathrm{U}_{q}\left(\mathfrak{p}_{-}\right)^{-}$. Thus $\mathcal{R}_{m}^{[n]}$ forms a right $\mathrm{U}_{q}\left(\mathfrak{g l}_{m}\right)$-module under the action $\tilde{\Psi}$. The $\Phi\left(\mathrm{U}_{q}\left(\mathfrak{g l}_{n}\right)\right)$ and $\tilde{\Psi}\left(\mathrm{U}_{q}\left(\mathfrak{g l}_{m}\right)\right)$ clearly preserve the algebraic structure of $\mathcal{R}_{m}^{[n]}$ in the sense of Definition 3.1. This completes the proof of part (1).

Now we prove the decomposition (3.1). This requires some preparation. Let $L_{\lambda}^{(0)}$ be an irreducible finite-dimensional $\mathrm{U}_{q}\left(\mathfrak{p}_{-}\right)$-module with highest weight $\lambda$. Then 
$L_{\lambda}^{(0)}$ is irreducible with respect to $\mathrm{U}_{q}\left(\mathfrak{g l}_{m}\right) \subset \mathrm{U}_{q}\left(\mathfrak{p}_{-}\right)$, and the elements of $\mathrm{U}_{q}\left(\mathfrak{p}_{-}\right)^{-}$ act on $L_{\lambda}^{(0)}$ by zero. Let

$$
\mathcal{O}_{\lambda}:=\left\{\zeta \in L_{\lambda}^{(0)} \otimes \mathcal{T}_{q}\left(\mathfrak{g l}_{n}\right) \mid\left(\mathrm{id} \otimes \Psi_{p}\right) \zeta=(S(p) \otimes \mathrm{id}) \zeta, \forall p \in \mathrm{U}_{q}\left(\mathfrak{p}_{-}\right)\right\} .
$$

Proposition 3.1. $\mathcal{O}_{\lambda}$ forms a left $\mathrm{U}_{q}\left(\mathfrak{g l}_{n}\right)$-module under the action

$$
\text { and } \mathcal{O}_{\lambda} \cong \begin{cases}L_{\lambda}, & \lambda \in \Lambda_{n}, \\ 0, & \text { otherwise. }\end{cases}
$$

Remark. Proposition [3.1 is the quantum Borel-Weil theorem [APW, PW, GZ] in our context. It can be easily deduced [GZ from the Peter-Weyl theory for $\mathcal{T}_{q}\left(\mathfrak{g l}_{n}\right)$, in particular, Proposition [2.1.

Now we turn back to the proof of (3.1). Consider the $\Phi \otimes \Psi$ action of $\mathrm{U}_{q}\left(\mathfrak{g l}_{n}\right) \otimes$ $\mathrm{U}_{q}\left(\mathfrak{g l}_{m}\right)$ on $\mathcal{R}_{m}^{[n]}$. One can always decompose $\mathcal{R}_{m}^{[n]}$ into

$$
\mathcal{R}_{m}^{[n]} \cong \bigoplus_{\lambda \in \Lambda_{m}} m_{\lambda} L_{\mu(\lambda)} \otimes\left(L_{\lambda}^{(0)}\right)^{*}
$$

where $\left(L_{\lambda}^{(0)}\right)^{*}$ is the dual vector space of the left $\mathrm{U}_{q}\left(\mathfrak{g l}_{m}\right)$-module $L_{\lambda}^{(0)}$, which has a natural left $\mathrm{U}_{q}\left(\mathfrak{g l}_{m}\right)$-module structure. The $m_{\lambda} \in \mathbb{Z}_{+}$is the multiplicity of the irreducible $\mathrm{U}_{q}\left(\mathfrak{g l}_{n}\right) \otimes \mathrm{U}_{q}\left(\mathfrak{g l}_{m}\right)$-module $L_{\mu(\lambda)} \otimes\left(L_{\lambda}^{(0)}\right)^{*}$ in $\mathcal{R}_{m}^{[n]}$, and $\mu(\lambda) \in \Lambda_{n}$ depends on $\lambda$. Take an irreducible $\mathrm{U}_{q}\left(\mathfrak{g l}_{m}\right)$-module $L_{\nu}^{(0)}, \nu \in \Lambda_{m}$, which can be extended to a unique $\mathrm{U}_{q}\left(\mathfrak{p}_{-}\right)$-module with $K_{c}, c>m$, acting by the identity map, and $E_{c c-1}, c>m$, acting by zero. We can therefore construct an $\mathcal{O}_{\nu}$ from $L_{\nu}^{(0)}$. It is easy to see that

$$
\mathcal{O}_{\nu}=\left(L_{\nu}^{(0)} \otimes \mathcal{R}_{m}^{[n]}\right)^{\mathrm{U}_{q}\left(\mathfrak{g l}_{m}\right)}
$$

where the $\mathrm{U}_{q}\left(\mathfrak{g l}_{m}\right)$-action is defined by $x(v \otimes f)=\sum_{(x)} x_{(1)} v \otimes \Psi_{x_{(2)}}(f)$, for all $x \in \mathrm{U}_{q}\left(\mathfrak{g l}_{m}\right), v \otimes f \in L_{\nu}^{(0)} \otimes \mathcal{R}_{m}^{[n]}$. The Borel-Weil Theorem (Proposition 3.1) forces

$$
m_{\lambda}=1, \quad \mu(\lambda)=\lambda, \quad \forall \lambda \in \Lambda_{m} .
$$

Thus with respect to $\Phi\left(\mathrm{U}_{q}\left(\mathfrak{g l}_{n}\right)\right) \otimes \Psi\left(\mathrm{U}_{q}\left(\mathfrak{g l}_{m}\right)\right)$,

$$
\mathcal{R}_{m}^{[n]} \cong \bigoplus_{\lambda \in \Lambda_{m}} L_{\lambda} \otimes\left(L_{\lambda}^{(0)}\right)^{*}
$$

This is equivalent to (3.1) with respect to $\Phi\left(\mathrm{U}_{q}\left(\mathfrak{g l}_{n}\right)\right) \otimes \tilde{\Psi}\left(\mathrm{U}_{q}\left(\mathfrak{g l}_{m}\right)\right)$ since the antipode $S$ of $\mathrm{U}_{q}\left(\mathfrak{g l}_{n}\right)$ is invertible.

The decomposition (3.1) implies that $\Phi\left(\mathrm{U}_{q}\left(\mathfrak{g l}_{n}\right)\right)$ and $\tilde{\Psi}\left(\mathrm{U}_{q}\left(\mathfrak{g l}_{m}\right)\right)$ are mutual centralizers in $\operatorname{End}_{\mathbb{C}(t)}\left(\mathcal{R}_{m}^{[n]}\right)$.

The $m=n$ case of the theorem leads to

Corollary 3.1. As a $\mathrm{U}_{q}\left(\mathfrak{g l}_{n}\right) \otimes \mathrm{U}_{q}\left(\mathfrak{g l}_{n}\right)$-module under the joint action $\Phi \otimes \tilde{\Psi}$,

$$
\mathcal{T}_{q}\left(\mathfrak{g l}_{n}\right) \cong \bigoplus_{\lambda \in \Lambda_{n}} L_{\lambda} \otimes \tilde{L}_{\lambda}
$$

This is also an immediate consequence of Proposition 2.1. 


\section{Definition 3.3.}

$$
\mathcal{L}_{m}^{[n]}:=\left\{f \in \mathcal{T}_{q}\left(\mathfrak{g l}_{n}\right) \mid \Phi_{K_{c}^{ \pm 1}}(f)=f, c>m, \Phi_{x}(f)=0, x \in \mathrm{U}_{q}\left(\mathfrak{p}_{+}\right)^{+}\right\} .
$$

Similar to the discussions on $\mathcal{R}_{m}^{[n]}$, we have the following results.

Lemma 3.2. The following set forms a basis of $\mathcal{L}_{m}^{[n]}$ :

$$
\left\{\prod_{1 \leq i \leq m, 1 \leq b \leq n}^{>}\left(t_{b i}\right)^{k_{b i}} \mid k_{b i} \in \mathbb{Z}_{+}\right\} .
$$

Theorem 3.2. (1) $\mathcal{L}_{m}^{[n]}$ forms a left module algebra over $\mathrm{U}_{q}\left(\mathfrak{g l}_{m}\right)$ under the action $\Phi$, and also forms a right module algebra over $\mathrm{U}_{q}\left(\mathfrak{g l}_{n}\right)$ under the action $\tilde{\Psi}$.

(2) With respect to the joint action $\Phi \otimes \tilde{\Psi}$ of $\mathrm{U}_{q}\left(\mathfrak{g l}_{m}\right) \otimes \mathrm{U}_{q}\left(\mathfrak{g l}_{n}\right)$,

$$
\mathcal{L}_{m}^{[n]} \cong \bigoplus_{\lambda \in \Lambda_{m}} L_{\lambda}^{(0)} \otimes \tilde{L}_{\lambda}
$$

The theorem can be proven in a way similar to the proof of Theorem 3.1. The following result will be of crucial importance. An irreducible $\mathrm{U}_{q}\left(\mathfrak{g l}_{m}\right)$-module $L_{\lambda}^{(0)}$ with highest weight $\lambda$ extends to a unique $\mathrm{U}_{q}\left(\mathfrak{p}_{+}\right)$-module with $\mathrm{U}_{q}\left(\mathfrak{p}_{+}\right)^{+}$acting by zero. Let $\tilde{\lambda}$ denote the lowest weight of $L_{\lambda}^{(0)}$. Define

$$
\tilde{\mathcal{O}}_{\lambda}:=\left\{\zeta \in L_{\lambda}^{(0)} \otimes \mathcal{T}_{q}\left(\mathfrak{g l}_{n}\right) \mid\left(\mathrm{id} \otimes \Phi_{p}\right) \zeta=(S(p) \otimes \mathrm{id}) \zeta, \forall p \in \mathrm{U}_{q}\left(\mathfrak{p}_{+}\right)\right\} .
$$

Proposition 3.2. $\tilde{\mathcal{O}}_{\lambda}$ forms a right $\mathrm{U}_{q}\left(\mathfrak{g l}_{n}\right)$-module under the action

$$
\begin{aligned}
& \qquad \tilde{\mathcal{O}}_{\lambda} \otimes \mathrm{U}_{q}\left(\mathfrak{g l}_{n}\right) \rightarrow \tilde{\mathcal{O}}_{\lambda}, \quad x \otimes \zeta \mapsto\left(\mathrm{id} \otimes \tilde{\Psi}_{x}\right) \zeta, \\
& \text { and } \tilde{\mathcal{O}}_{\lambda} \cong \begin{cases}\tilde{L}_{-\tilde{\lambda}}, & -\tilde{\lambda} \in \Lambda_{n}, \\
0, & \text { otherwise. }\end{cases}
\end{aligned}
$$

Remark. This is a variant of the quantum Borel-Weil theorem [APW, PW, GZ] and easily follows [GZ] from Proposition 2.1.

\section{Definition 3.4.}

$$
\mathcal{V}_{m, n}:= \begin{cases}\mathcal{R}_{m}^{[n]}, & m \leq n, \\ \mathcal{L}_{n}^{[m]}, & m \geq n .\end{cases}
$$

Combining Theorems 3.1 and 3.2 we arrive at the quantum Howe duality, Theorem 1.1.

\section{Howe duality implies Schur Duality}

We adapt the definition of the $q$-Weyl group of $[\mathrm{KR}$ to the Jimbo setting of quantized enveloping algebras. Denote by $\mathcal{M}$ the set of finite-dimensional $\mathrm{U}_{q}\left(\mathfrak{g l}_{n}\right)$ modules of type I. Every $M \in \mathcal{M}$ is semisimple, i.e., $M=\bigoplus L_{\lambda} \otimes M_{\lambda}$. Let $\operatorname{Aut}(\mathcal{M})$ be the set consisting of the automorphisms of the underlying $\mathbb{C}(t)$-vector spaces of all the elements of $\mathcal{M}$. Let $\mathcal{G}$ be the set of the maps $\sigma: \mathcal{M} \rightarrow \operatorname{Aut}(\mathcal{M})$ such that $\sigma(M): M \rightarrow M$, and are of the form

$$
\sigma(M)=\sum \sigma\left(L_{\lambda}\right) \otimes \operatorname{id}_{M_{\lambda}}
$$


for any $M=\bigoplus L_{\lambda} \otimes M_{\lambda} \in \mathcal{M}$. Therefore, a $\sigma \in \mathcal{G}$ is uniquely determined once $\sigma\left(L_{\lambda}\right)$ are given for all $L_{\lambda} \in \mathcal{M}$. Clearly, the diagram

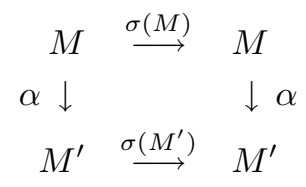

commutes for any morphism $\alpha: M \rightarrow M^{\prime}$ of finite-dimensional $\mathrm{U}_{q}\left(\mathfrak{g l}_{n}\right)$-modules of type I. Furthermore, $\mathcal{G}$ forms a group, with the multiplication $\left(\sigma, \sigma^{\prime}\right) \mapsto \sigma \sigma^{\prime}$ and inverse map $\sigma \mapsto \sigma^{-1}$, respectively, defined by $\left(\sigma \sigma^{\prime}\right)(M)=\sigma(M) \sigma^{\prime}(M)$, and $\sigma^{-1}(M)=(\sigma(M))^{-1}$, for all $M \in \mathcal{M}$. The identity of this group is the map $M \mapsto \operatorname{id}_{M}, \forall M$. The $q$-Weyl group $\mathcal{W}_{q}(n)$ of $\mathrm{U}_{q}\left(\mathfrak{g l}_{n}\right)$ is a subgroup of $\mathcal{G}$ which we now define.

Let $U^{(j, c)}$ be the type I irreducible $\mathrm{U}_{q}\left(\mathfrak{g l}_{2}\right)$-module with the highest weight $(j+c) \epsilon_{1}+(j-c) \epsilon_{2} \in \mathbb{Z} \epsilon_{1} \oplus \mathbb{Z} \epsilon_{2}$ such that $j \in \mathbb{Z}_{+} / 2$. Consider a basis $\left\{u_{m}^{(j, c)} \mid\right.$ $m=j, j-1, \ldots, 1-j,-j\}$ of $U^{(j, c)}$ defined by

$$
\begin{aligned}
K_{1} u_{m}^{(j, c)} & =q^{c+m} u_{m}^{(j, c)}, \\
K_{2} u_{m}^{(j, c)} & =q^{c-m} u_{m}^{(j, c)}, \\
E_{1} u_{m}^{(j, c)} & =[j+m+1][j-m] u_{m+1}^{(j, c)}, \\
F_{1} u_{m}^{(j, c)} & =u_{m-1}^{(j, c)},
\end{aligned}
$$

where $[j]=\frac{q^{j}-q^{-j}}{q-q^{-1}}$. (Recall that $q=t^{2}$.) Define an automorphism $w^{(j, c)}$ of the underlying vector space of $U^{(j, c)}$ by

$$
w^{(j, c)} u_{m}^{(j, c)}=(-1)^{j-m} t^{-2 j(j+1)-2 c^{2}} \frac{[j-m]}{[j+m]} u_{-m}^{(j, c)} .
$$

Remark. Let $\mathcal{A}=\mathbb{C}\left[t, t^{-1}\right]$ be the subring of $\mathbb{C}(t)$ consisting of the Laurent polynomials in $t$. Then $w^{(j, c)}$ defines an automorphism of the free $\mathcal{A}$-module $U_{\mathcal{A}}^{(j, c)}$ with basis $\left\{u_{m}^{(j, c)}\right\}$.

Let $\mathrm{U}_{q}^{a}\left(\mathfrak{g l}_{2}\right)$ be the subalgebra of $\mathrm{U}_{q}\left(\mathfrak{g l}_{n}\right)$ generated by $E_{a}, F_{a}, K_{a}^{ \pm 1}$ and $K_{a+1}^{ \pm 1}$, for a fixed $a<n$. Corresponding to every $\mathrm{U}_{q}^{a}\left(\mathfrak{g l}_{2}\right)$, we define a $w_{a} \in \mathcal{G}$ in the following way. Any irreducible $\mathrm{U}_{q}\left(\mathfrak{g l}_{n}\right)$-module $L_{\lambda} \in \mathcal{M}$ restricts to a semisimple $\mathrm{U}_{q}^{a}\left(\mathfrak{g l}_{2}\right)$-module, $L_{\lambda}=\bigoplus U^{(j, c)} \otimes Z_{(j, c)}^{\lambda}$, where $U^{(j, c)}$ is the irreducible $\mathrm{U}_{q}^{a}\left(\mathfrak{g l}_{2}\right)$ module defined by (4.2). We define $w_{a}\left(L_{\lambda}\right):=\sum w^{(j, c)} \otimes \mathrm{id}_{Z_{(j, c)}^{\lambda}}$, and extend $w_{a}$ to an element of $\mathcal{G}$ through (4.1).

Definition 4.1. The $q$-Weyl group $\mathcal{W}_{q}(n)$ of $\mathrm{U}_{q}\left(\mathfrak{g l}_{n}\right)$ is the subgroup of $\mathcal{G}$ generated by the $w_{a}$ and $\left(w_{a}\right)^{-1}, a=1,2, \ldots, n-1$.

Remark. $\mathcal{W}_{q}(n)$ is a slight modification of the $q$-Weyl group of $\mathrm{U}_{q}\left(\mathfrak{s l}_{n}\right)$ given in [KR] because of the inclusion of $c$ in [4.3). Both $q$-Weyl groups have essentially the same properties.

Remark. A $q$-Weyl group can also be defined with respect to right $\mathrm{U}_{q}\left(\mathfrak{g l}_{n}\right)$-modules in the obvious way. Results parallel to Theorem 4.1 and Proposition 4.1 below hold for the right module version of the $q$-Weyl group. 
For a finite dimensional type $\mathrm{I} \mathrm{U}_{q}\left(\mathfrak{g l}_{n}\right)$-module $M$, we define

$$
(M)_{0}:=\left\{v \in M \mid\left(K_{a}-q\right) v=0, \forall a\right\},
$$

and call it the space of zero weight vectors of $M$. Clearly $(M)_{0}$ is stable under all the $w_{a}(M)$, thus forms a $\mathcal{W}_{q}(n)$-submodule of $M$. Since each finite-dimensional $\mathrm{U}_{q}\left(\mathfrak{g l}_{n}\right)$-module of type I is isomorphic to the tensor product of a one-dimensional $\mathrm{U}_{q}\left(\mathfrak{g l}_{n}\right)$-module with an $L_{\lambda}, \lambda \in \Lambda_{n}$, the study of the $\mathcal{W}_{q}(n)$-modules $(M)_{0}$ essentially reduces to studying $\left(V^{\otimes k}\right)_{0}, k \in \mathbb{Z}_{+}$, where $V$ is the contravariant vector $\mathrm{U}_{q}\left(\mathfrak{g l}_{n}\right)$-module. Note that $\left(V^{\otimes k}\right)_{0}=0$ unless $k=n$.

The $\mathbb{C}(t)$-space $\left(V^{\otimes n}\right)_{0}$ has a basis $\left\{v_{\sigma(1)} \otimes v_{\sigma(2)} \otimes \ldots \otimes v_{\sigma(n)} \mid \sigma \in \mathfrak{S}_{n}\right\}$, where $\mathfrak{S}_{n}$ is the symmetric group on $n$ letters. Denote the action of the $q$-Weyl group on $\left(V^{\otimes n}\right)_{0}$ by $\tau: \mathcal{W}_{q}(n) \times\left(V^{\otimes n}\right)_{0} \rightarrow\left(V^{\otimes n}\right)_{0}$.

Theorem 4.1. $\mathbb{C}(t) \tau\left(\mathcal{W}_{q}(n)\right)$ is a homomorphic image of the Hecke algebra $\mathcal{H}_{a}(n)$. More explicitly,

$$
\begin{aligned}
\tau\left(w_{a}\right) \tau\left(w_{b}\right) & =\tau\left(w_{b}\right) \tau\left(w_{a}\right),|a-b|>1, \\
\tau\left(w_{a}\right) \tau\left(w_{a+1}\right) \tau\left(w_{a}\right) & =\tau\left(w_{a+1}\right) \tau\left(w_{a}\right) \tau\left(w_{a+1}\right), \\
\left(q^{2} \tau\left(w_{a}\right)-q\right)\left(q^{2} \tau\left(w_{a}\right)+q^{-1}\right) & =0, \forall a .
\end{aligned}
$$

Proof. It can be easily deduced from results of $\mathrm{KR}$, (or from the formulae (4.7) below) that the $w_{a}$ obey the relations of the braid group of type $A$. Thus we only need to prove the quadratic relations (4.6). Fix an index $a$; then $\left(V^{\otimes n}\right)_{0}$ is spanned by the zero weight vectors of copies of the $\mathrm{U}_{q}^{a}\left(\mathfrak{g l}_{2}\right)$-modules $U^{(1,1)}$ and $U^{(0,1)}$. Acting on $\left(U^{(1,1)}\right)_{0}$ and $\left(U^{(0,1)}\right)_{0}, w_{a}$ takes eigenvalues $-q^{-3}$ and $q^{-1}$ respectively. Hence the quadratic relations (4.6).

We can describe the action of $\mathcal{W}_{q}(n)$ on $\left(V^{\otimes n}\right)_{0}$ more explicitly. Consider vectors $\zeta_{a}, \eta_{a} \in\left(V^{\otimes n}\right)_{0}$ of the form $\zeta_{a}:=X \otimes v_{a} \otimes Y \otimes v_{a+1} \otimes Z$ and $\eta_{a}:=X \otimes v_{a+1} \otimes$ $Y \otimes v_{a} \otimes Z$. We have

$$
\begin{aligned}
q^{2} \tau\left(w_{a}\right)\left(\zeta_{a}\right) & =\left(q-q^{-1}\right) \zeta_{a}-\eta_{a}, \\
q^{2} \tau\left(w_{a}\right)\left(\eta_{a}\right) & =-\zeta_{a} .
\end{aligned}
$$

Remark. Using the formulae (4.7) one can easily prove Theorem 4.1 by direct computations.

When $q$ is specialized to 1 , the action of $\mathcal{W}_{q}(n)$ on $\left(V^{\otimes n}\right)_{0}$ reduces to that of the symmetric group $\mathfrak{S}_{n}$. To explain this in more precise terms, we consider the free module $V_{\mathcal{A}}$ over $\mathcal{A}\left(\mathcal{A}=\mathbb{C}\left[t, t^{-1}\right]\right)$ generated by the elements of the standard basis $\left\{v_{a} \mid a=1,2, \ldots, n\right\}$ of $V$. Denote by $V_{\mathcal{A}}^{\otimes n}$ the $n$-th power of $V_{\mathcal{A}}$ over $\mathcal{A}$. Set $\left(V_{\mathcal{A}}^{\otimes n}\right)_{0}:=\left\{w \in V_{\mathcal{A}}^{\otimes n} \mid\left(K_{a}-q\right) w=0, \forall a\right\}$, which is a free $\mathcal{A}$-module. Because of the remark following equation (4.3), $\left(V_{\mathcal{A}}^{\otimes n}\right)_{0}$ is invariant under $\mathcal{W}_{q}(n)$. Introduce to $\mathbb{C}$ the following $\mathcal{A}$-module structure: $\psi: \mathcal{A} \otimes_{\mathbb{C}} \mathbb{C} \rightarrow \mathbb{C}, a(t) \otimes 1 \mapsto a(1)$. We define $\mathcal{A} \mathcal{W}_{q}(n) \otimes_{\mathcal{A}} \mathbb{C}$ and $\left(V_{\mathcal{A}}^{\otimes n}\right)_{0} \otimes_{\mathcal{A}} \mathbb{C}$ using $\psi$, and consider the action of $\mathcal{A} \mathcal{W}_{q}(n) \otimes_{\mathcal{A}} \mathbb{C}$ on $\left(V_{\mathcal{A}}^{\otimes n}\right)_{0} \otimes_{\mathcal{A}} \mathbb{C}$. Let $i_{k}, k=1,2, \ldots, n$, be distinct elements of $\{1,2, \ldots, n\}$. We have

$$
-w_{a} \otimes 1: v_{i_{1}} \otimes v_{i_{2}} \otimes \ldots \otimes v_{i_{n}} \otimes 1 \mapsto v_{s_{a}\left(i_{1}\right)} \otimes v_{s_{a}\left(i_{2}\right)} \otimes \ldots \otimes v_{s_{a}\left(i_{n}\right)} \otimes 1,
$$

where $s_{a} \in \mathfrak{S}_{n}$ permutes $a$ and $a+1$ while leaving the other elements of $\{1,2, \ldots, n\}$ unchanged. 
Proposition 4.1. Assume that $L_{\lambda}$ is an irreducible $\mathrm{U}_{q}\left(\mathfrak{g l}_{n}\right)$-module of type I with highest weight $\lambda \in \Lambda_{n}(n)$. Then the space of zero weight vectors $\left(L_{\lambda}\right)_{0}$ forms the irreducible $\mathcal{H}_{q}(n)$-module determined by the partition corresponding to $\lambda$. Also, every irreducible $\mathcal{H}_{q}(n)$-module is isomorphic to an $\left(L_{\lambda}\right)_{0}$ with $\lambda \in \Lambda_{n}(n)$.

Proof. Since any type I irreducible $\mathrm{U}_{q}\left(\mathfrak{g l}_{n}\right)$-module $L_{\lambda}$ with highest weight $\lambda \in$ $\Lambda_{n}(n)$ can be embedded into $V^{\otimes n}$, and this is also an embedding of $\mathbb{C}(t) \mathcal{W}_{q}(n)$ modules, we only need to consider the case when $L_{\lambda}$ is an irreducible $\mathrm{U}_{q}\left(\mathfrak{g l}_{n}\right)$ submodule of $V^{\otimes n}$. Let $L_{\lambda, \mathcal{A}}:=V_{\mathcal{A}}^{\otimes n} \cap L_{\lambda}$, and $\left(L_{\lambda, \mathcal{A}}\right)_{0}:=V_{\mathcal{A}}^{\otimes n} \cap\left(L_{\lambda}\right)_{0}$, where $V_{\mathcal{A}}^{\otimes n}$ is regarded as a subset of $V^{\otimes n}$. Now we specialize $t$ to 1 by using $\psi$. It follows from results of Lusztig and Rosso (see [CP]) on quantized enveloping algebras at generic $q$ that

$$
\begin{aligned}
L_{\lambda, \mathcal{A}} \otimes_{\mathcal{A}} \mathbb{C} & \cong L_{\lambda}^{0}, \\
\left(L_{\lambda, \mathcal{A}}\right)_{0} \otimes_{\mathcal{A}} \mathbb{C} & \cong\left(L_{\lambda}^{0}\right)_{0},
\end{aligned}
$$

where $L_{\lambda}^{0}$ is the irreducible $\mathfrak{g l}_{n}$-module with highest weight $\lambda$, and $\left(L_{\lambda}^{0}\right)_{0}$ is the space of zero weight vectors of $L_{\lambda}^{0}$. It is known that $\left(L_{\lambda}^{0}\right)_{0}$ forms the irreducible $\mathfrak{S}_{n}$-module characterized by the partition corresponding to $\lambda$. (This result is due to Schur.

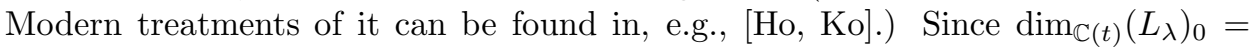
$\operatorname{dim}_{\mathbb{C}}\left(L_{\lambda}^{0}\right)_{0}$, and any $\mathcal{H}_{q}(n)$-submodule of $\left(L_{\lambda}\right)_{0}$ would give rise to an $\mathfrak{S}_{n}$-submodule of $\left(L_{\lambda}^{0}\right)_{0}$, we conclude that $\left(L_{\lambda}\right)_{0}$ is irreducible with respect to $\mathcal{H}_{q}(n)$. By recalling the fact (see $\mathrm{Ma}$ ) that $\mathcal{H}_{q}(n)$ and $\mathbb{C}(t) \mathfrak{S}_{n}$ are isomorphic as associative algebras and are semisimple, we easily see that $\left(L_{\lambda}\right)_{0}$ is the irreducible left $\mathcal{H}_{q}(n)$-module $S_{\lambda}$ determined by the partition corresponding to $\lambda$. The $S_{\lambda}, \forall \lambda \in \Lambda_{n}(n)$, exhaust all the irreducible $\mathcal{H}_{q}(n)$-modules up to isomorphisms.

We now derive from Theorem 1.1 Jimbo's quantum Schur duality at generic $q$ [Ji], which amounts to the following statement.

Theorem 4.2. Let $V$ be the contravariant vector module over $\mathrm{U}_{q}\left(\mathfrak{g l}_{n}\right)$. Then

$$
V^{\otimes m}=\bigoplus_{\lambda \in \Lambda_{n}(m)} L_{\lambda} \otimes \tilde{M}_{\lambda},
$$

where $\tilde{M}_{\lambda}$ is the irreducible right $\mathcal{H}_{q}(m)$-module associated with $\lambda$.

Proof. We shall follow the same strategy as that of section 2.4.5.2 in [Ho]. Consider the space $\left(\mathcal{V}_{n, m}\right)_{0, \mathrm{U}_{q}\left(\mathfrak{g l}_{m}\right)}$ of zero $\mathrm{U}_{q}\left(\mathfrak{g l}_{m}\right)$-weight vectors of $\mathcal{V}_{n, m}$ under the right action of $\mathrm{U}_{q}\left(\mathfrak{g l}_{m}\right)$. As a left $\mathrm{U}_{q}\left(\mathfrak{g l}_{n}\right)$-module, $\left(\mathcal{V}_{n, m}\right)_{0, \mathrm{U}_{q}\left(\mathfrak{g l}_{m}\right)}$ is isomorphic to $V^{\otimes m}$. Following Theorem 1.1.

$$
V^{\otimes m}=\bigoplus_{\lambda \in \Lambda_{n}(m)} L_{\lambda}^{[n]} \otimes\left(\tilde{L}_{\lambda}^{[m]}\right)_{0},
$$

where we have used the fact that $\left(\tilde{L}_{\lambda}^{[m]}\right)_{0} \neq 0$ if and only if $|\lambda|=m$. The right module version of Proposition 4.1 immediately leads to what we seek to prove.

\section{SOME REMARKS}

Our proof of Theorem 1.1 makes essential use of the Peter-Weyl basis (Proposition 2.1) of $\mathcal{T}_{q}\left(\mathfrak{g l}_{n}\right)$ via Propositions 3.1 and 3.2. When $q$ is specialized to a root of unity, Proposition 2.1 fails completely. However, $\mathcal{T}_{q}\left(\mathfrak{g l}_{n}\right)$ is still well defined [PW], and so is also $\mathcal{V}_{m, n}$. It is clearly true that $\mathcal{V}_{m, n}$ forms a module algebra under the 
left action $\Phi$ of $\mathrm{U}_{q}\left(\mathfrak{g l}_{m}\right)$ and right action $\tilde{\Psi}$ of $\mathrm{U}_{q}\left(\mathfrak{g l}_{n}\right)$, with the actions of the two quantum algebras commuting. Then a natural question is whether the following relations hold at roots of unity:

$$
\begin{aligned}
& \left.\Phi\left(\mathrm{U}_{q}\left(\mathfrak{g l}_{m}\right)\right)\right|_{\mathcal{V}_{m, n}}=\operatorname{End}_{\mathrm{U}_{\mathrm{q}}\left(\mathfrak{g l} \mathfrak{l}_{\mathrm{n}}\right)}\left(\mathcal{V}_{\mathrm{m}, \mathrm{n}}\right), \\
& \left.\tilde{\Psi}\left(\mathrm{U}_{q}\left(\mathfrak{g l}_{n}\right)\right)\right|_{\mathcal{V}_{m, n}}=\operatorname{End}_{\mathrm{U}_{\mathrm{q}}\left(\mathfrak{g l}_{\mathrm{m}}\right)}\left(\mathcal{V}_{\mathrm{m}, \mathrm{n}}\right) .
\end{aligned}
$$

It is quite likely that the answer is affirmative in view of the fact [PW] that the actions of $\mathrm{U}_{q}\left(\mathfrak{g l}_{n}\right)$ and $\mathcal{H}_{q}(m)$ on $V^{\otimes m}$ are centralizers of each other in $\operatorname{End}_{\mathbb{C}}\left(\mathrm{V}^{\otimes \mathrm{m}}\right)$ even at roots of unity.

\section{REFERENCES}

[APW] Andersen, H. H.; Polo, P.; Wen, K. X., Representations of quantum algebras. Invent. Math. 104 (1991) 1-59. MR 92e:17011

[CP] Chari, V.; Pressley, A., A guide to quantum groups. Cambridge University Press, Cambridge, 1994. MR 95j:17010

[GZ] Gover, A. R.; Zhang, R. B., Geometry of quantum homogeneous vector bundles and representation theory of quantum groups. I. Rev. Math. Phys. 11 (1999) 533-552. MR 2000j:81108

[Ho] Howe, R., Perspectives on invariant theory. The Schur Lectures (1992). Eds. I. PiatetskiShapiro and S. Gelbart, Bar-Ilan University, 1995. MR 96e:13006

[Ji] Jimbo, M., Quantum $R$ matrix related to the generalized Toda system: an algebraic approach. In Field theory, quantum gravity and strings (Meudon/Paris, 1984/1985), 335-361, Lecture Notes in Phys., 246, Springer, Berlin (1986). MR 87j:17013

[Ko] Kostant, B., On Macdonald's $\eta$-function formula, the Laplacian and generalized exponents, Adv. Math. 20 (1976) 257-285. MR 58:5484

[KR] Kirillov, A. N., Reshetikhin, N., q-Weyl group and a multiplicative formula for universal $R$-matrices. Comm. Math. Phys. 134 (1990) 421-431. MR 92c:17023

[Ma] Mathas, A., Iwahori-Hecke algebras and Schur algebras of the symmetric group. Providence, R.I. : American Mathematical Society (1999). MR 2001g:20006

[Mon] Montgomery, S., Hopf algebras and their actions on rings. CBMS Regional Conference Series in Math., 82. American Mathematical Society, Providence, RI, 1993. MR 94i:16019

[PW] Parshall, B.; Wang, J. P., Quantum linear groups. Mem. Amer. Math. Soc. 89 (1991), no. 439. MR 91g:16028

[FRT] Reshetikhin, N. Yu.; Takhtadzhyan, L. A.; Faddeev, L. D., Quantization of Lie groups and Lie algebras. Algebra i Analiz 1 (1989) 178-206. (Russian) MR 90j:17039

[Ta] Takeuchi, M., Some topics on $\mathrm{GL}_{q}(n)$. J. Algebra 147 (1992) 379-410. MR 93b:17055

School of Mathematics and Statistics, University of Sydney, Sydney, New South Wales 2006, Australia

E-mail address: rzhang@maths.usyd.edu.au 